\title{
Invisible Radiations of Organisms
}

\section{Invisible Radiations of Organisms}

By Prof. Otto Rahn. With an Introduction to the Physics of Radiation, by Sidney W. Barnes. (Protoplasma-Monographien, Vol. 9.) Pp. $x+215$. (Berlin: Gebrüder Borntraeger, 1936.) 13.20 gold marks.

$\mathrm{H}$

ERE we behold mitogenetic radiation masquerading as the legitimate offspring of biochemistry and the quantum theory, and the unwary reader, finding 'muscle radiation' casually mentioned in a purely physical account of the photo-electric effect, may well suppose the scientific status of the two phenomena to be identical. On reaching p. 55 he may begin to suspect that in reality mitogenetic radiation was quite differently conceived; but up to this point the emission of such radiation by living cells, and even by simple systems undergoing chemical reaction, is treated as a predictable phenomenon related to chemiluminescence, and is described with the same confidence as the dispersion of light by a prism. Even the too remarkable phenomena of 'secondary radiation', involving emission of fluorescent radiation of shorter wave-length and higher intensity than the incident radiation, and its propagation, by successive phases of absorption and re-emission, through an absorbing medium, are made to appear almost as inevitable as Newton's laws of motion.

From p. 55 onwards, apart from a fair discussion of the physical detection of mitogenetic radiation, the book is biological, dealing largely with material which has already been adequately presented by Gurwitsch in his two monographs : the biological detection, phenomenology and significance of mitogenetic radiation. In addition to this, there are a few references to more recent work, accounts of experiments by the author and his collaborators, and some rather uncritical remarks about the alleged lethal effects of menstrual blood on yeast and bacteria.

A brief epilogue is devoted to the question of errors in the detection of mitogenetic radiation and to the attitude of those who remain unconvinced of its existence. Piously hoping for a rapprochement between believers and non-believers, Prof. Rahn does not seem to realize that his book is not likely to help in the attainment of that object. The critics ask only that a detalled reexamination of the original mitogenetic effect shall be undertaken by some of those who claim to be able to obtain positive results without difficulty; but Prof. Rahn and his Russian colleagues prefer to write redundant monographs on the ubiquity of mitogenetic radiation, to discuss it in relation to amphibian metamorphosis, parasitism, polyploidy in tomato plants, industrial fatigue, cancer and old age, and to invent queer chain reactions to hide its physico-chemical misbehaviour.

\section{Properties of Iron}

\section{The Metal-Iron}

By H. E. Cleaves and J. G. Thompson. (Alloys of Iron Research Monograph Series.) (Published for the Engineering Foundation.) Pp. xii +574 . (New York and London: McGraw-Hill Book Co., Inc., 1935.) 36s. net.

TO the valuable series of monographs on the 1 alloys of iron, issued by the Engineering Foundation, there has now been added a volume dealing with the production and properties of the parent metal in a state approaching purity. Although iron is used in far larger quantities in industry than any other metal, and is not obtainable commercially in such a pure condition as several other metals, such as aluminium and zinc, our knowledge of its exact properties is still very imperfect. There are two main reasons for this fact. With its high melting point and great chemical activity, iron readily absorbs impurities when in the molten state, which are correspondingly difficult to remove, whilst its properties are exceptionally influenced by the presence of even very small quantities of foreign elements. The properties assigned to 'pure' iron in the text-books are mostly derived from commercial materials regarded as approximately pure, or by extrapolation from a series of samples with diminishing amounts of impurity. The results are in either case unsatisfactory.

The present work, by two members of the staff of the U.S. Bureau of Standards, covers the 